Session 2793

\title{
Interactive Multimedia Intelligent Tutoring System (IMITS) : A Progress Report
}

\author{
Brian P. Butz, Michael Duarte \\ Intelligent Systems Application Laboratory \\ College of Engineering \\ Temple University \\ Philadelphia, PA 19122
}

\begin{abstract}
The Interactive Multimedia Intelligent Tutoring System (IMITS) is an intelligent tutoring system that is focussed on the instruction of undergraduate electrical engineering students. The material covered is that typically presented in courses on introductory circuits. At present, it consists of three modules: DC, AC and Transient Analysis. IMITS is intended as a course supplement. While there is a substantial amount of multimedia material and interactions, the software package is intended as a tutor, providing extra help for a student taking a face-toface, on-line, or self-directed course. As the student progresses through the material, the expert system that is embedded in the software will watch and analyze the student's activities. By doing this, it is able to determine the student's strengths and weaknesses and determine what material should be presented in what order. The IMITS software environment has been under development for three years. It has recently been sent to several colleges and universities throughout the country for testing and evaluation. This paper discusses the present state of IMITS.
\end{abstract}

Introduction

A continual challenge in teaching is meeting the students' academic needs and ensuring that they are active participants in their own education. Over the last decade, studies ${ }^{1-5}$ have reinforced the notion that students best learn if they are active participants in their education. Interactive multimedia software is playing a key and unique role in the educational process. It has been used as a stand-alone educational module that is intended to enable an interested individual to learn about a particular topic or subject ${ }^{6}$, as a supplement to classroom presentations ${ }^{7-9}$, and to laboratories ${ }^{10,11}$, and as a dynamic textbook ${ }^{12}$. Recently, interactive multimedia have been integrated with expert system technology producing intelligent tutoring systems for engineering students ${ }^{13-16}$. These intelligent tutoring systems "learn" about the individual student and can tailor the material to meet the needs and the learning styles appropriate to the individual student.

The goal of the Interactive Multimedia Intelligent Tutoring System (IMITS) is to develop an intelligent tutor for an introductory sequence in electrical circuits and systems. The framework developed for this system is to be transferable to and used by any discipline, not only engineering. The project has as its major goals to:

1) Develop and integrate the components necessary to form an interactive multimedia intelligent tutoring system. 
2) Develop the interactive multimedia materials necessary to supplement a two semester introductory course on electric circuits for electrical engineering undergraduates and a twosemester course in electric circuits and systems for engineering students not majoring in electrical engineering.

3) Develop a simulated laboratory that will enable students to select, place and interconnect various circuit elements, electronic devices, electrical systems, signal generators and measuring instruments.

4) Permit the student to connect in any physically feasible way the elements and instruments available in a typical laboratory storeroom and obtain the same responses, including failures, that would be experienced in the laboratory.

5) Simulate the performance of the interconnected elements and objects by integrating them seamlessly with software application packages. These packages, such as PSpice, will produce a data stream representing current, voltages, frequencies, power, etc. and will be used as inputs to the various simulated measuring instruments.

6) Provide the student with an intelligent laboratory assistant that will answer the student's questions in a way that simulates the role of a laboratory technician who is expert in the subject matter.

7) Evaluate the effectiveness of the intelligent tutoring system and describe its affect on student learning.

8) Place the resultant modules on CD ROMS and make these modules completely functional on the World Wide Web.

9) Disseminate the results over the World Wide Web, in appropriate journals and through presentation at notable conferences.

The project outcome will be a self-contained software package that will enable students taking introductory electrical circuits and systems courses receive tutoring directed by an intelligent agent. The interactive multimedia courseware will be contained in six modules: DC Analysis, Transient Analysis, AC Analysis, Devices, Digital Systems, and Analog Systems. The DC, AC and Transient Analysis modules will be used in the two introductory circuits courses for electrical engineering undergraduate students. Parts of these and the other modules will be used in the two survey courses on the principles electrical engineering.

During development, it quickly became apparent that the presentation of the material must not only be pedagogically sound and rich but also be engaging. To be engaging, it must allow the student a flexibility that is unusual in intelligent tutoring system development. To accomplish these objectives a story line was adopted so that the student could play a role and make choices allowing him/her to choose how to solve assignments. The story line chosen was that of a junior engineer newly hired by the multinational IMITS Corporation. The new engineer works in an office (Figure 1) and selects a design team to join. The design team the student selects specializes in the area in which the student desires tutoring. While in the office a student receives a televideo message from the virtual supervisor. In the message, the supervisor explains a new assignment that the student is receiving. The assignment involves understanding electrical engineering concepts that have been covered up to that point in the course. Working on the assignment requires critical thinking skills because the student has to understand it, plan how to complete the assignment and choose among the concepts learned to select those that will assist in solving the assignment. 


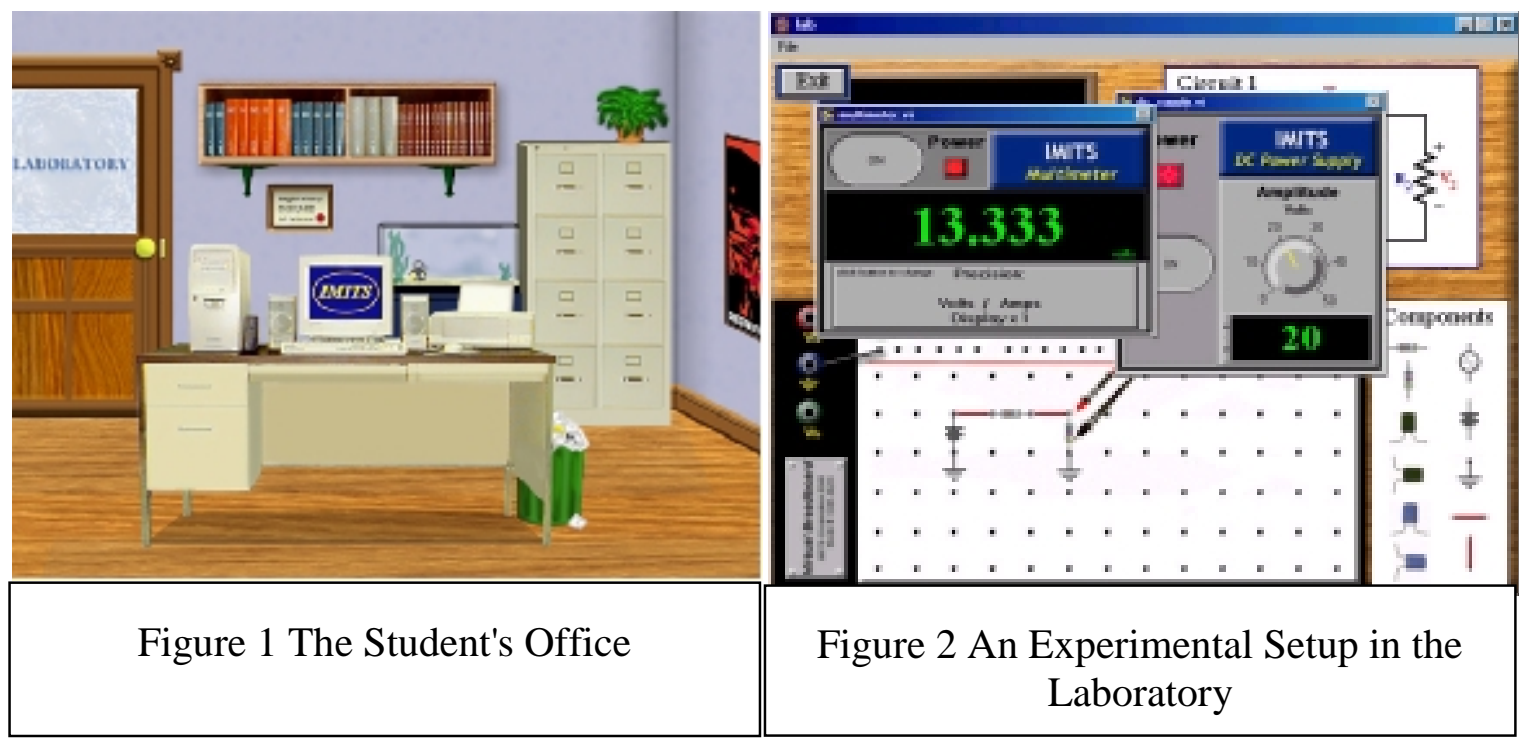

Once the assignment is made, the student may complete the assignment using any of the resources available. Resources include books containing explanations of material needed to perform the assignment. This expository material is highly interactive and asks the students many questions. Each question is asked for a specific reason and has a learning objective associated with it. Questions may be multiple choice, short answer, or drag and drop. Additional resources consist of file cabinet drawers containing corporation notes and specifications about various corporation products. The student interacts directly with the virtual team leader and, if needed, the virtual skills advancement director. The student also has access, through a door in the office, to a virtual laboratory (Figure 2) where the student may build and test possible designs. In addition, the IMITS Corporation provides some internal training on its own using case studies that serve as assignments.

When the student feels that s/he knows the solution, s/he emails it back to the supervisor. The supervisor acknowledges that the assignment solution is either correct or offers additional help to solve the assignment. A description of the IMITS learning mechanism has been given previously ${ }^{14}$ and will not be repeated here.

\section{Project Status}

Three of the six modules, DC Analysis, AC Analysis and Transient Analysis have been completed. Consequently, the intelligent tutoring system is able to assist students in their first two courses in circuit theory. The assignment is the basis of learning within the IMITS software package. The assignments have to be done in a linear fashion-one assignment completed or mastered before the next assignment may be undertaken. More than just the correct answer for the assignment is required of the student. Even if the student completes the assignment correctly, using none of the interactive and questioning features available, specific questions await the student at the end of the assignment. The so-called "objective questions" are based on the learning objectives of the assignment and ensure that the student knows how to do the assignment. Thus, the student is evaluated based on his/her knowledge of the learning objectives, not the correct completion of an assignment. The assignment sequence is used to build the tutor so it sequentially presents material that is more difficult. 
There are six assignments within each of the three modules, or eighteen assignments in all. The student may use books on the bookshelf to review material that will help the student complete each assignment. Three books are pertinent to the software developed thus far: DC Analysis, AC Analysis and Transient Analysis. The contents of each book are shown in Figures 3 through 5.

\begin{tabular}{|l}
\hline Chapter 1 Introduction \\
$1.1 \quad$ What is an Electric Circuit? \\
$1.2 \quad$ Current and Voltage \\
$1.3 \quad$ Power \\
Chapter 2 Kirchoff's Laws \\
$2.1 \quad$ Kirchoff's Current Law \\
$2.2 \quad$ Kirchoff's Voltage Law \\
Chapter 3 Sources \\
Chapter 4 Resistive Circuits \\
$4.1 \quad$ Resistance and Ohm's Law \\
$4.2 \quad$ Voltage and Current Dividers \\
Chapter 5 Nodal and Mesh Analysis \\
$5.1 \quad$ Node Voltage Analysis \\
$5.2 \quad$ Mesh Current Analysis \\
Chapter 6 Equivalent Circuits \\
Chapter 7 Thevenin Equivalent Circuit
\end{tabular}

Figure 3. Contents of DC Analysis Book

Introduction
Circuit Example
Motor Example
Chapter 1 Time Constants
Chapter 2 Initial Conditions
Chapter 3 Inductors and Capacitors
Chapter 4 Step Response
Chapter $\mathbf{5}$ First Order Systems
Types of Response
$5.1 \quad$ Natural Response
$5.2 \quad$ Forced Response
$5.3 \quad$ Forced Response to a Constant Input
$5.4 \quad$ Forced Response to a Time Varying Input
$5.5 \quad$ Complete Response
Chapter 6 Second Order Systems
$6.1 \quad$ Introduction
$6.2 \quad$ Natural Response
$6.3 \quad$ Applying Initial Conditions
$6.4 \quad$ Forced Response
$6.5 \quad$ Complete Response

Figure 4. Contents of Transient Analysis Book 


\section{Chapter 1 Phase and Phasors}

1.1 Sinusoidal Review

1.2 What are Phasors?

1.3 Manipulating Phasors

Chapter 2 Impedance

Chapter 3 Impedance and Frequency

Chapter 4 Frequency Response

4.1 Introduction

4.2 Transfer Functions

4.3 Bode Plot Derivation

4.4 Bode Plot Approximation

4.5 Filters

Chapter 5 AC Power

5.1 Energy and Power

5.2 Instantaneous and Average Power

5.3 Effective Power

5.4 Instantaneous and Average Power

5.5 Effective Power

Chapter 6 Transforms

6.1 Laplace Transforms

6.2 Inverse Laplace Transforms

Chapter 7 Demonstrations

7.1 Experimental Determination of Frequency Response

7.2 Power Plant

Figure 5. Contents of AC Analysis Book

As with a printed text, any chapter or subchapter may be read by the student in any order desired.

Now and then the student is referred to company drawings, specifications, reports and notices that are found in the file cabinet. The student opens the file cabinet drawer, finds the corresponding letter of the document and clicks on that letter's folder to see the appropriate information. The computer monitor, when clicked upon, yields the screen shown in Figure 6. Clicking on Scratch Pad gives the student access to his or her notebook where notes or data may be kept. If the student clicks on "calculator the standard Microsoft Windows ${ }^{\circledR}$ scientific calculator appears. Clicking on "email" allows the student to receive or send virtual email. Email is sent from the student's supervisor to give the necessary details of a student's assignment. Once the student devises a solution to the assignment, it is sent to the supervisor via email. Clicking on "teleconferencing" allows the student to receive a video message from the supervisor. The student is informed that this message exists by the sound of a ringing telephone. When the student clicks on the computer monitor icon in the office, and then on the teleconferencing icon, a video message is played. The video message might be a straightforward message from the supervisor announcing the problem and describing how assignment particulars may be obtained. The video might also be a vignette that sets up the problem. 


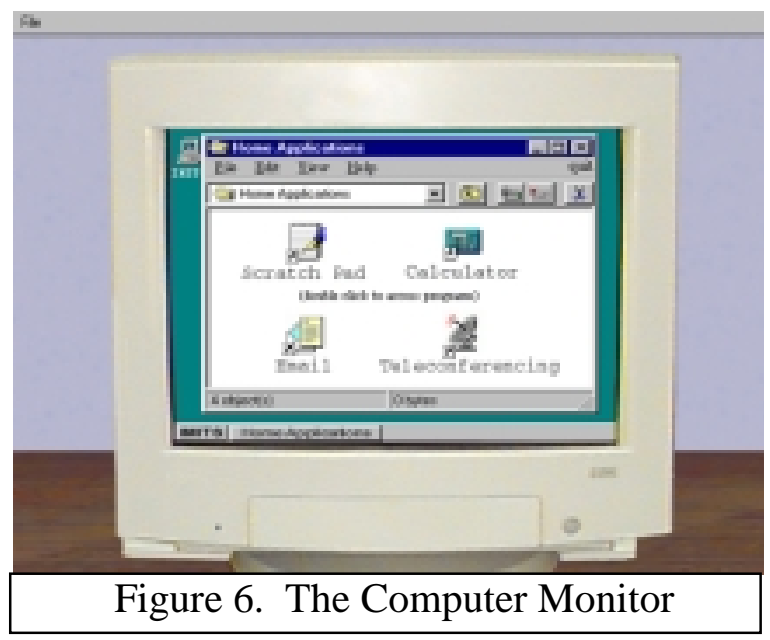

The student may visit the virtual laboratory by clicking on the lab door from the office. The Virtual Laboratory consists of a breadboard, components, and miniature instruments. Figure 2 shows a typical experimental setup. The Virtual Laboratory users can build and test circuitry with the following components: resistors, capacitors, inductors, and jumper wires. In addition, the laboratory consists of the following instruments: a DC power supply, function generator, multimeter, oscilloscope, and spectrum analyzer with frequency sweep generator.

As described in reference 14, the expert system watches the student progress through the assignments. If the student performs poorly on any of the software's 36 learning objectives ${ }^{1}$, the expert system takes appropriate action, sending the student to the appropriate chapter in the appropriate book or providing direct tutoring.

Testing and Evaluation

During the Spring 2002 semester the IMITS software package will be beta-tested and evaluated within the electrical engineering or similar departments at the following schools: Drexel University, Duke University, Howard University, Montgomery County Community College (PA), New Mexico State University, and Temple University. The spring 2002 evaluation has two objectives: The first objective is to test and evaluate the software design and usability. An assessment will be made of how easy is the software to use. Students and instructors will be asked to evaluate the software. Answers will be sought to the questions: How easy is the software to use? How useful is it? Is the content sufficient? Is the content accurate and well presented? What other features, not present, are desired? What modifications should be made? A questionnaire has been developed to obtain this information and will be web-based. At the end of the semester, students and faculty will be asked to complete the questionnaire. In addition, the students will be asked to keep a running diary throughout the semester and it is planned to have weekly virtual conferences with all the instructors participating in the test.

The second objective is broader in scope. It focuses on individual differences in use of the software. We have identified three characteristics that might result in different approaches/strategies to using the software: (a) cognitive style (b) spatial ability, and (c) learning style. Each of these can be assessed by paper and pencil instruments that will be administered to

\footnotetext{
${ }^{1}$ A learning objective is a basic concept that forms an essential body of knowledge in the area being studied. For example, within electrical engineering, the understanding of Kirchoff's voltage (KVL) and current laws (KCL) is essential to understanding the foundation of circuit theory and KCL/KVL is designated a learning objective.
} 
the students as a group. As students use the software, various files are created that reflect dependent variables of interest. We will then compare differences in usage of the software based on the individual differences noted above. Questions include does a student follow software paths that coincide with the student's perceived learning preference? Are learning preferences static or dynamic? How do field dependent students use the software? How do field independent students use the software?

The software environment, developed using Macromedia's Authorware, allows us to record detailed data that describes the student's usage of the software. For example, we can trace where the student goes in the environment. We will know what submodules a student visits, when they are visited, and how long the student remains within a submodule. We can determine if a student asks for hints as well as what submodules the student chooses not to visit. Also, we update and record the student's scores for each learning objective. We also record the student's more recent score for each learning objective. All data is recorded within three student files for each student. In order to retrieve the files at the end of the evaluation, we will ask that students upload files to our website at the end of the semester. Dr. Susan Miller of Temple's Educational Psychology Department will play an essential role in developing a detailed evaluation protocol for analyzing and making sense of the recorded data.

The spring 2002 evaluation is a prelude to the overall evaluation effort planned during the 200203 academic year. By fall 2002, the software will have been thoroughly tested and necessary revisions will be made. The set of dependent variables that will satisfy our second objective mentioned above will be identified. We will attempt to measure the effectiveness of this software and try to quantify its contribution to student learning.

Ellis ${ }^{17}$ has noted how difficult it is to provide concrete evidence of multimedia value. He notes that there are two major obstacles:

"First it is difficult to define and measure learning. There is little agreement concerning what constitutes learning and exactly how to measure its presence and extent." 17

Further,

"Second, it is difficult to identify comparable instructional tools.

To compare the effectiveness of multimedia as an instructional tool, one must compare the results of students using that tool with the results of students using a comparable, non-multimedia tool. Comparing an excellent textbook with a poorly designed and implemented computer-aided instruction system would not produce meaningful results.",17

One might add that comparing excellent interactive multimedia courseware with a poor textbook or classroom technique likewise would not produce meaningful results.

Based on the results and analyses of the evaluation data and the resulting revisions of the software package, we will develop a detailed protocol for determining the effectiveness of the software. 
Conclusions

This paper discusses an intelligent tutoring system that has been developed for tutoring electrical engineering students in introductory circuit theory and analysis. The system, IMITS, is unique because the software is project centered and learner directed. The content consists of material covered in a two-semester circuits course. The framework developed for IMITS is transferable to almost any academic material and currently the framework is being applied to a business course and for health education.

During the spring, 2002 semester the software package will be beta-tested at six institutions of higher learning. The evaluation will measure the software' usability and the richness and validity of its content. The data recorded during the evaluation will give us a preliminary assessment of our ability to ascertain different approaches and strategies employed by students in using the software. This will assist in developing a comprehensive protocol to measure the effectiveness of the software.

For updated information about the IMITS project and other intelligent tutoring systems we are developing, visit our website at http://www.temple.edu/IMITS.

Acknowledgements

Partial support for this work was provided by the National Science Foundation's Division of Undergraduate Education through grant DUE \#9952291.

Bibliographic Information

1. Felder, R. (1996). Matters of Style. ASEE Prism, 6 (4), 18-23.

2. Lumsdaine, M., and Lumsdaine, E. (1987). Thinking Preferences of Engineering Students: Implications for Curriculum Restructuring. Journal of Engineering Education, 84 (2), 193-204.

3. Stice, J.E. (1987). Using Kolb's Learning Cycle to Improve Student Learning. Engineering Education, 77, 291-296.

4. Felder, R.M., and Silverman, L.K. (1988). Learning and Teaching Styles in Engineering Education, Engineering Education, 78 (7), 674-681.

5. Carver, C.A. Jr. et al (1999). Enhancing Student Learning Through Hypermedia Courseware and Incorporation of Student Learning Styles, IEEE Transactions on Education, 42 (1), 33-38.

6. Sears, A.L., and Watkins, S.E. (1996). A Multimedia Manual on the World Wide Web for Telecommunications Equipment. IEEE Transactions on Education, 39 (3), 342-348.

7. Butz, B.P. (1997). Interactive Multimedia Software for a Course in Introductory Circuits. Proceedings of the AACE World Conference on Educational Multimedia and Hypermedia, Calgary, Alberta, Canada.

8. Edminister, J.A. (1995). Electric Circuits, Schaum's Interactive Online Series, New York, NY, McGraw-Hill, Inc

9. DiStefano, J.J., Stubberud, A.R., and Williams, I.J. (1995). Feedback and Control Systems, Schaum's Interactive Outline Series, New York, NY, McGraw-Hill, Inc

10. Mosterman, P.J. et al (1996). Design and Implementation of an Electronics Laboratory Simulator. IEEE Transactions on Education, 39 (3), 327-335.

11. Bergland, M. et al (1999). Case It! A Project to Integrate Collaborative Case-Based Learning into International Undergraduate Biology Curricula. Selected Papers from the 10th International Conference on College Teaching and Learning (J. A. Chambers Ed.), Jacksonville, FL, 1-8.

12. Harger, R.O. (1996). Teaching in a Computer Classroom with a Hyperlinked Interactive Book. IEEE Transactions on Education, 39 (3), 327-335. 
13. Butz, B.P. (1999). An Interactive Multimedia Intelligent Tutoring System, Selected Papers from the 10th International Conference on College Teaching and Learning (J. A. Chambers Ed.), Jacksonville, FL, 15-22.

14. Butz, B.P. (2001). The Learning Mechanism of the Interactive Multimedia Intelligent Tutoring System (IMITS), Engineering Education, 90 (4), 543-548.

15. Tambe, M. et al, (1995). Intelligent Agents for Interactive Simulation Environments, AI Magazine, 16 (1), 1539.

16. Milton-Benoit, J. et al (1998). The Multimedia Finite Element Modeling and Analysis Tutor, Journal of Engineering Education, 87 (5), 511-517.

17. Ellis, T.J., (2001). Multimedia Enhanced Educational Products as a TOOL to Promote Critical Thinking in Adult Students, Jl. Of Educational Multimedia and Hypermedia, 10 (2), 107-123.

\section{Biographical information}

Dr. BRIAN P. BUTZ is a Professor of Electrical and Computer Engineering at Temple University, Philadelphia, PA. He is the Director of the Intelligent Systems Application Laboratory (ISAL) which provides a focal point within Temple University concentrating on research in intelligent systems. Since 1997, he has been working on intelligent tutoring systems.

MICHAEL DUARTE is a Senior Software Applications Engineer working in the Intelligent Systems Application Laboratory (ISAL) at Temple University. Since the fall of 1997, he has been one of the lead designers of the Interactive Multimedia Intelligent Tutoring System (IMITS) and the Universal Virtual Laboratory (UVL), two of the most highly funded projects within ISAL. He received his Masters Degree in Electrical Engineering in 2001. 